\title{
REACTIVITE THERMIQUE DE LA PEAU SCROTALE CHEZ L'HOMME
}

\author{
F. Becmeur*, B. Bothorel*, A. Clavert**, V. Candas* \\ * L.P.P.E., CNRS/INRS, 21, Rue Becquerel, Strasbourg \\ **CECOS Alsace, Hôpitaux universitaires de Strasbourg
}

\section{THERMAL REACTIVITY OF THE SCROTAL SKIN IN MAN. Although the} existence of heat exchange between the testicular artery and the adjacent veins is well known, it may be insufficient to maintain the lower temperature of the testis. In order to investigate the role of the scrotum in testicular thermoregulation, the dynamics of scrotal and other skin temperatures was studied in relation to thermal stress. The results of this study revealed that the scrotal skin exhibited a greater thermal inertia than did other skin areas. This finding may be due to modifications in scrotal blood flow patterns as temperature increased, as well as changes in the scrotal surface area. Other possible thermoregulatory factors may include the evaporation of sweat from the scrotum. In conclusion, there seems to be a complex collection of mechanisms, rather than a single specific mechanism, regulating testicular temperature. Key words : Scrotum, testicular thermoregulation. Andrologie, 1992, 2 : 48-49.

L'urologue anglais Griffith (in 2), semble avoir été le premier en 1893 à reconnaître que la descente du testicule en position extra-abdominale est une condition "sine qua non " du développement normal des fonctions du testicule chez l'homme. Pour Crew dès 1922 (in 2), la raison en était un problème de température. Depuis lors on sait que le maintien du testicule à une température de l'ordre de $34^{\circ} \mathrm{C}$ est indispensable pour que l'histologie de la gonade et la spermatogénèse soient normales. La thermolyse du testicule par le système à contre-courant de la vascularisation cordonale au niveau du plexus pampiniforme est connue $(1,5)$. Reste à découvrir comment se font les échanges thermiques avec l'extérieur. Peu d'expériences connues décrivent l'élévation des températures scrotales en réaction à la contrainte thermique chez l'homme sain: la plus récente a été faite dans des conditions spéciales incluant la climatisation des membres inférieurs (1). Les transferts de chaleur du testicule au milieu extérieur peuvent ils être assurés par le scrotum ? Amorçant l'étude de la physiologie scrotale chez l'homme, nous avons comparé l'évolution des températures cutanées sur l'ensemble du corps avec celle des températures scrotales, lors de contraintes thermiques.

\section{MATERIEL ET METHODES}

L'étude s'est déroulée au Laboratoire de Physiologie et Psychologie Environnementales du C.N.R.S. à Strasbourg, dans une chambre climatique: la température (Ta) de l'air ambiant, sa vitesse, et son hygrométrie peuvent être régulées, ainsi que la température des parois . Après accord du comité d'éthique local, nous avons recruté 5 sujets mâles entre 20 et 25 ans. Il s'agissait de sujets sains, sans antécédents. Une thermographie de contact à cristaux liquides a permis d'éliminer toute hyperthermie scrotale. Alors que le sujet reposait sur un hamac dans une chambre climatique,les températures scrotales droite et gauche ont été enregistrées à l'aide de capteurs de température dont la thermistance était en contact avec la peau, maintenue par deux ailettes latérales collées par un ruban adhésif non allergénique. De la même façon, 10 températures cutanées, dont la température cutanée abdominale et la température cutanée thoracique étaient mesurées. La température cutanée moyenne, Tsk, est la température pondérée au prorata des surfaces de chacune des 10 mesures précédentes.

Tableau 1 : Conditions imposées au cours des trois expériences $\mathrm{E} 1, \mathrm{E} 2, \mathrm{E} 3$. Les conditions $\mathrm{E} 2$ et $\mathrm{E} 3$ comportent chacune deux phases incluant ou non l'activité physique.

$W=$ exercice physique à bras (soulever à deux bras un poids de $4 \mathrm{~kg}, 24$ fois par minute, sujet allongé sur un hamac). Ta = température de l'air circulant dans la chambre climatique. Tw $=$ température des parois de la chambre climatique. $\mathrm{T}_{0}=$ tem . pérature opérative (ambiante).

\begin{tabular}{lccccc}
\hline & E1 & \multicolumn{2}{c}{ E2 } & \multicolumn{2}{c}{ E3 } \\
\hline W & non & non & oui & oui & non \\
\hline Ta & 34 & 28 & 28 & 28 & 28 \\
Tw & 69 & 69 & 69 & 28 & 69 \\
To & 46 & 43 & 43 & 28 & 43 \\
\hline
\end{tabular}

Chaque sujet a été soumis à 3 conditions expérimentales différentes (E1, E2, E3) décrites dans le tableau 1 . Une période de thermoneutralité a été réalisée durant les 30 premières minutes de chaque expérience. Une contrainte thermique externe a été imposée durant au moins 60 minutes à des moments différents au cours de chaque expérience. Il s'agissait d'élever les tem- pératures de l'air ambiant (Ta) circulant dans la chambre climatique, ainsi que la température des parois de la pièce (Tw), l'ensemble de ces modifications thermiques réalisant une température opérative aux alentours de $45^{\circ} \mathrm{C}$. La température opérative est une moyenne pondérée par les coefficients d'échange de chaleur des Ta et Tw.

Figure 1 : Températures mesurées pendant les expériences.

TSK $=$ moyenne des 10 températures cutanées mesurées sur l'ensemble du corps hormis le scrotum. $\mathrm{ABDO}=$ température cutanée de la paroi abdominale (une des mesures pour le calcul de TSK). Test. Droit et Test. Gauche = températures cutanées scrotales.

De 0 à 15 minutes $=$ repos. De 20 à 60 minutes $=$ exercice physique. De 60 à 140 minutes $=$ repos mais élévation des température de la chambre climatique.

$\mathrm{NB}=$ l'exercice physique n'a pas modifié les températures cutanées, soit par insuffisance d'effort physique, soit par l'effet de ventilation secondaire aux mouvements dus sujet allongé nu sur un hamac.
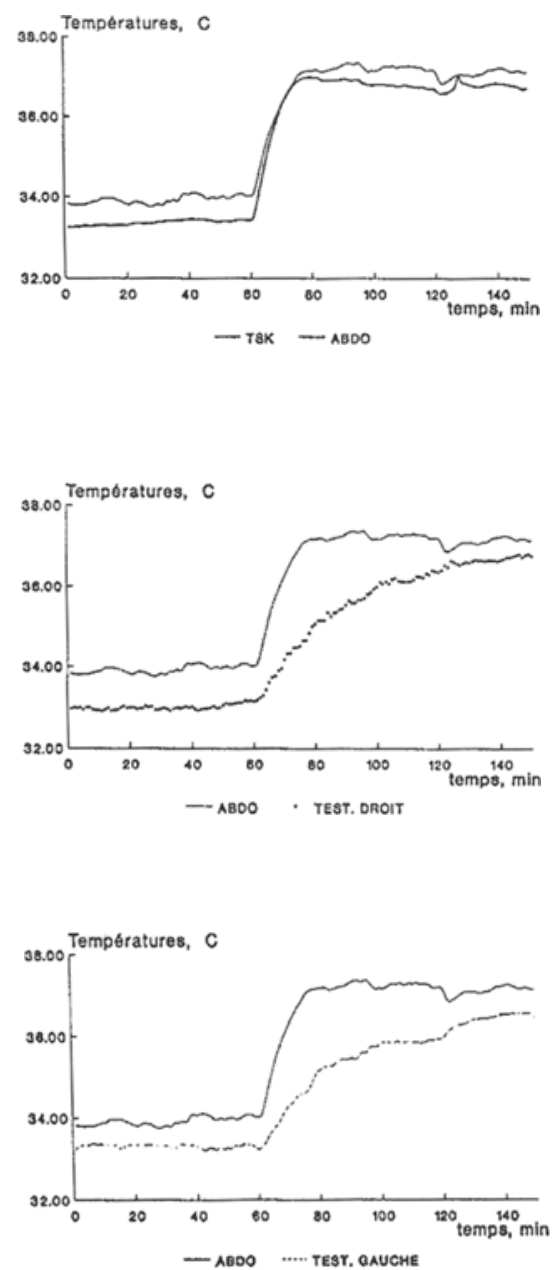
Une contrainte thermique interne par exercice physique à deux bras, levant et abaissant un poids de $4 \mathrm{~kg}, 24$ fois par minute, a été menée durant 45 minutes après les 60 premières minutes d'exposition au chaud pour E2 (I'environnement étant inchangé), ou avant les 60 minutes de chaleur en E3 (voir tableau 1).

\section{RESULTATS}

Les résultats moyens des températures cutanées des 5 sujets exposés à la condition E3 sont donnés dans la figure 1. Durant la phase de thermoneutralité, la température oesophagienne moyenne est de $36^{\circ} \mathrm{C}+1-0,2^{\circ} \mathrm{C}$. La température cutanée moyenne est de $33^{\circ} 4 \mathrm{C}+1-0,3^{\circ} \mathrm{C}$. La différence de température d'environ $3^{\circ} \mathrm{C}$ s'explique par la nécessité d'éliminer la chaleur endogène métabolique. La température cutanée scrotale moyenne est de $33^{\circ} 4 \mathrm{C}+1-0,4^{\circ} \mathrm{C}$. Les températures du scrotum à droite et à gauche évoluent de façon semblable, mais leur décours montre qu'elles n'évoluent pas comme une température de peau normale abdominale ou cutanée moyenne. L'inertie thermique est en effet supérieure, ce qui évite l'échauffement rapide même si en fin de contrainte thermique chaude, les températures scrotales sont presque aussi élevées que sur les autres zones cutanées. La peau scrotale se réchauffe, mais moins vite que les autres territoires cutanés examinés.

\section{DISCUSSION}

L'inertie thermique scrotale peut en partie être expliquée par les propriétés vasomotrices du scrotum. On sait que l'élévation de la température scrotale accroit le flux sanguin local dans des proportions considérables (6). Mais le scrotum a de nombreuses particularités: la peau est fine, dépourvue de graisse sous cutanée, et sous-tendue par un muscle peaucier appelé dartos, dont les contractions peuvent faire varier la surface scrotale d'environ $20 \%$. Sa vascularisation est connue, mais certaines caractéristiques de l'axe honteux externe mériteraient une étude expérimentale. L' étude de l'innervation du scrotum et des thermorécepteurs mérite aussi de nouvelles recherches. Les caractéristiques évaporatoires du scrotum font l'objet d'études en cours et complèteront l'analyse du bilan thermolytique local.

\section{CONCLUSION}

Il n'existe vraisemblablement pas de thermorégulation exclusivement spécifique du testicule, mais un ensemble d'éléments ou un " complexe thermolytique " aboutissant à un effet d'amortissement thermique dans les limites quantitatives ou temporelles de contrainte thermique modérée. Le scrotum apparaît bien, comme l'affirme Mieusset (3), être un des deux éléments essentiels de la régulation thermique du testicule. L'autre élément, situé entre l'abdomen et le testicule, serait constitué du plexus pampiniforme.

\section{REFERENCES}

1 - Dewasmes G., Bothorel B., Hsiung R., Clavert A., Candas V.: Human scrotal temperature during heat exposure associated with passive leg heating. In : Temperature Environmental Effects on the Testis. AW Zorgniotti. Ed. New-York, 1989, 286: 187-192.

2 - Harrison R.G., Weiner J.S. : Vascular patterns of mamalian testis and their functional significance. J. Exp. Biol., 1949, 26 : 304-316.

3- Knaus H : Die physiologische Bedentung des Scotums. Klin. Wochenschr., 1932, 2: 1897-1900.

4 - Mieusset R : Régulation thermique de la fonction testiculaire. Rech. Gynecol., 1989, 3: 163-171.

5 - Setchell B.P. : The mamalian testis. The scrotum and thermoregulation. P. Elek éd., London, 1978.

6 - Waites G.H.M., Setchell B.P., Quinlan D : Effects of local heating of the scrotum testes and epididymis of rats on cardiac output and regional blood flow. J. Reprod. Fert., 1973, $34: 41-49$.

RESUME : La comparaison du décours des températures scrotales avec celui d'autres températures cutanées lors de contraintes thermiques témoigne d'une plus grande inertie thermique scrotale. Mots clés : Scrotum, thermorégulation du testitule. Andrologie, 1992,2 : $48-49$.

\section{INSTRUCTIONS AUX AUTEURS}

Les textes scientifiques publiés dans Andrologie doivent être constitués comme suit, et adressés en quatre exemplaires au rédacteur en chef :

1. Page de garde comportant titre, nom(s), prénom(s) et adresse(s) de(s) institution(s) de(s) auteur(s).

2. Page suivante comportant un titre anglais, un résumé anglais plus long et détaillé que le résumé français, mais ne devant pas dépasser 30 lignes dactylographiées double interligne, et une liste de 2 à 6 mots clés en anglais.

3. A partir de la page suivante, texte dactylographié en double interligne sur un seul côté de la page, et selon un format normalisé : 27 lignes de 60 caractères et espaces par ligne. Chaque fois que possible, la présentation doit suivre un plan classique, c'est à dire : Introduction, Matériel et méthodes, Résultats, Discussion, Références. Le texte doit être le plus concis possible.

4. Les références sont à présenter sous le titre "REFERENCES", et par ordre aiphabétique. Elles soivent être appelées dans le texte par leur numéro. Leur nombre doit être limité à 10 au maximum pour 4 pages de texte ( 20 pour 8 pages, 30 pour 12 pages, sauf cas particulier de revue générale). Suivre le modèle ci-après :

1 - Auroux M, Dulioust E. Cyclophosphamide in the male rat: Behavioral effects in the adults in artificial insemination. In : David G, Price WS eds. Human artificial insemination and semen preservation. New York, Plenum Press, 1980: 197-210.

2 - Schwartz D, Mayaux MJ. Mode of evaluation of results in artificial insemination. In : David G, Price WS eds. Human artificial insemination and semen preservation. New York, Plenum Press, 1980 : 197-210.

Lorsqu'il y a plus de six auteurs, ne citer que les 3 premiers, suivis de la mention "et al".

5. Sur la page suivant les références, placer un résumé en français court, maximum 10 lignes dactylographiées double interligne, suivi d'une liste de 2 à 6 mots clés en français.

6. Les tableaux et figures doivent être présentés à part, 1 par page. Les légendes des différentes figures doivent être dactylographiées sur une page séparée. Le numéro des figures doit être inscrit au dos de chacune, au crayon de bois. Les photos en noir et blanc peuvent être reproduites, mais pas les photos et illustrations en couleur. 